Laurita Martins de AZEVÊDO ${ }^{1}$

Maria Acelina Martins de CARVALHO

Danilo José Ayres de MENEZES 2

Gilberto Valente

$\mathrm{MACHADO}^{3}$

Antonio Augusto Rodrigues de SOUSA ${ }^{4}$

Fabiana Galtarossa XAVIER ${ }^{5}$

Correspondência para:

Departamento de Morfofisiologia Veterinária, Centro de Ciências Agrárias da Universidade Federal do Piauí. Campus da Socopo, sem número. CEP 64049-550 Teresina/Piauí - mcelina@ufpi.br / carvalhomam@uol.com.br

Telefone/fax: + 558632155748

Recebido para publicação: 28/07/06 Aprovado para publicação: 25/10/07

\title{
Distribuição intraparenquimal da artéria hepática em cutias (Dasyprocta sp, Rodentia)
}

\author{
1 - Departamento de Morfofisiologia Veterinária do Centro de Ciências Agrárias da \\ Universidade Federal do Piauí, Teresina - PI \\ 2 - Unidade Acadêmica de Medicina Veterinária do Centro de Saúde e Tecnologia \\ Rural da Universidade Federal de Campina Grande, Patos - PB \\ 3 - Universidade Federal do Paraná, Palotina - PR \\ 4 - Universidade Estadual do Maranhão, São Luis - MA \\ 5 - Faculdade de Medicina Veterinária e Zootecnia da Universidade de São Paulo, \\ São Paulo - SP
}

\section{Resumo}

Com o objetivo de analisar a ramificação da artéria hepática e sua distribuição intraparenquimal em cutias, foram utilizados dez fígados de cutias adultas, fêmeas e machas, cedidas pelo Núcleo de Estudos e Preservação de Animais Silvestres do Centro de Ciências Agrárias da Universidade Federal do Piauí (Convênio FUFPI/IBAMA número 02/99). Oito fígados foram dissecados pela face visceral após injeção com látex do tipo Neoprene 650 (DuPont do Brasil Industrias Químicas) corado em vermelho, através da artéria hepática, e fixados em solução aquosa de formol a 10\%, durante pelo menos 48 horas. Dois órgãos foram utilizados para a confecção dos moldes vasculares, através de injeção de solução de acetato de vinil corado em vermelho e corrosão em solução aquosa de ácido clorídrico a 30\% até total destruição do parênquima. $\mathrm{O}$ estudo das peças mostrou que a artéria hepática na cutia divide-se em dois ramos principais, direito e esquerdo $(100 \%)$. De forma geral, o ramo direito predominantemente $(80 \%)$ origina vasos responsáveis pela vascularização dos lobos medial direito, lateral direito e caudado. O ramo esquerdo apresenta-se com maior freqüência $(80 \%)$ constituindo um tronco comum aos vasos que se destinam aos lobos lateral esquerdo, medial esquerdo, quadrado, em $50 \%$, também ao lobo caudado e em $20 \%$ ao lobo lateral direito. Sendo assim, conclui-se que a distribuição vascular da artéria hepática está relacionada à lobação do órgão, onde os ramos direito e esquerdo se distribuem no parênquima dos lobos do fígado, podendo-se inferir a presença de territórios com vascularização própria nos lobos hepáticos, caracterizando, portanto, a segmentação anátomocirúrgica arterial.

\section{Introdução}

Nos últimos anos, tem-se verificado no Brasil um notável incremento na criação de animais silvestres como nova alternativa para a produção de alimentos, principalmente nas regiões mais carentes. Nestes locais, a criação e exploração em cativeiro de animais nativos proporciona fonte de proteína animal de baixo custo e ainda colabora como medida de preservação ambiental destas espécies. ${ }^{1}$

Existe na fauna brasileira uma diversidade de espécies silvestres com potencial zootécnico pelas suas características favoráveis para a exploração econômica. Dentre estas, destaca-se a cutia (Danyprocta $s p)$, um mamífero roedor neotropical de porte médio, bastante adaptado às condições do semi-árido, com capacidade de se reproduzir em cativeiro, boa prolificidade, precocidade, domicilidade, período de 
gestação relativamente curto e outros atributos biológicos desejáveis à domesticação. ${ }^{2,3}$

Há poucos dados na literatura sobre as características morfofisiológicas dos diversos órgãos da cutia, incluindo a descrição do aporte arterial e venoso para os diversos sistemas. Pesquisas anteriores foram realizadas sobre a configuração do sistema venoso portal na cutia ${ }^{4}$ e distribuição intraparenquimal da veia porta hepática neste roedor ${ }^{5}$.

Considerando-se a artéria hepática, existem dados sobre sua origem e ramificação extraparenquimal na cutia ${ }^{6} \mathrm{e} \mathrm{em}$ animais de laboratório, como ratos e camundongos $^{7,8,9}$. Outros autores descrevem a sua distribuição em coelhos ${ }^{1}$ e caprinos. ${ }^{10}$

Ainda sobre a sistematização da artéria hepática, Souza, Souza e Miglino ${ }^{11}$ citam que, na capivara (Hydrochoerus hydrochaeris), este vaso origina em $66,6 \%$ dos animais dois outros ramos, sendo que um deles dirigesepara o lobo lateral esquerdo e segue, consecutivamente, para o lobo caudado, processo caudado, processo papilar e lobo lateral direito; o outro ramo destina-se ao lobo medial esquerdo, quadrado, medial direito e, finalmente, à vesícula biliar, onde origina a artéria cística. Em 33,3\%, os vasos apresentaram diferentes arranjos. Enquanto que em coelhos, Barone ${ }^{1}$ cita que da artéria hepática propriamente dita, se destaca, no hilo do fígado, um ramo para o lobo caudado e, em seguida, um ramo para o lobo direito, do qual se deriva a artéria cística. A artéria termina em dois ramos, sendo um para o lobo lateral esquerdo e o outro para o medial esquerdo. Este último, origina a artéria do lobo quadrado.

Neste sentido, diante da ausência dessas informações na cutia, o presente trabalho apresenta a distribuição arterial no parênquima hepático deste roedor como uma contribuição ao estudo da Anatomia dos animais silvestres de interesse zootécnico. De outro modo, apresentar bases anatômicas que favoreçam os avanços cirúrgicos, especialmente referentes às ressecções parciais do fígado.

\section{Material e Método}

Foram analisados 10 fígados de cutias adultas, fêmeas e machos, cedidos pelo Núcleo de Estudos de Preservação de Animais Silvestres do Centro de Ciências Agrárias da Universidade Federal do Piauí (Convênio FUFPI/IBAMA número 02/99). As peças foram obtidas a partir de animais que vieram à óbito naturalmente ou que foram submetidos a eutanásia após anestesia com a associação de acepromazina $(0,1 \mathrm{mg} /$ $\mathrm{kg}$, via intramuscular) com tiletaminazolazepam $(10 \mathrm{mg} / \mathrm{Kg}$, via intramuscular).

Após a abertura da cavidade abdominal e retirada do fígado, a artéria hepática foi canulada e perfundida com Neoprene látex 650 (DuPont do Brasil Industrias Quimicas), corado em vermelho, em oito peças. Em seguida, os órgãos foram fixadas em solução aquosa de formol a 10\%, durante um período mínimo de 48 horas, em superfície plana, sendo, posteriormente, dissecados pela face visceral do fígado. As peças dissecadas foram esquematizadas $\mathrm{e}$ fotografadas, com vistas à descrição dos resultados e ilustração da pesquisa.

Para a obtenção dos moldes vasculares, foram utilizados dois fígados, os quais receberam injeção de solução de acetato de vinil corado em vermelho e, em seguida, foram submetidos à técnica de corrosão em solução aquosa de ácido clorídrico a 30\%, até total destruição do parênquima do órgão.

\section{Resultados e Discussão}

Os resultados mostraram que a artéria hepática divide-se, em $90 \%$ dos animais, em dois ramos principais, o direito e o esquerdo (Figuras 1, 2 e3). A mesma divisão foi descrita para os caprinos ${ }^{10}$ e capivaras ${ }^{11}$.

No que concerne ao ramo direito da artéria hepática na cutia, presente em 100\% dos animais, este emitia em $80 \%$ das peças, vasos destinados aos lobos medial direito, lateral direito e caudado (Figura 3). Estes dados se assemelham aos descritos por Franco et al. ${ }^{10}$ em carneiros e por Barone et 


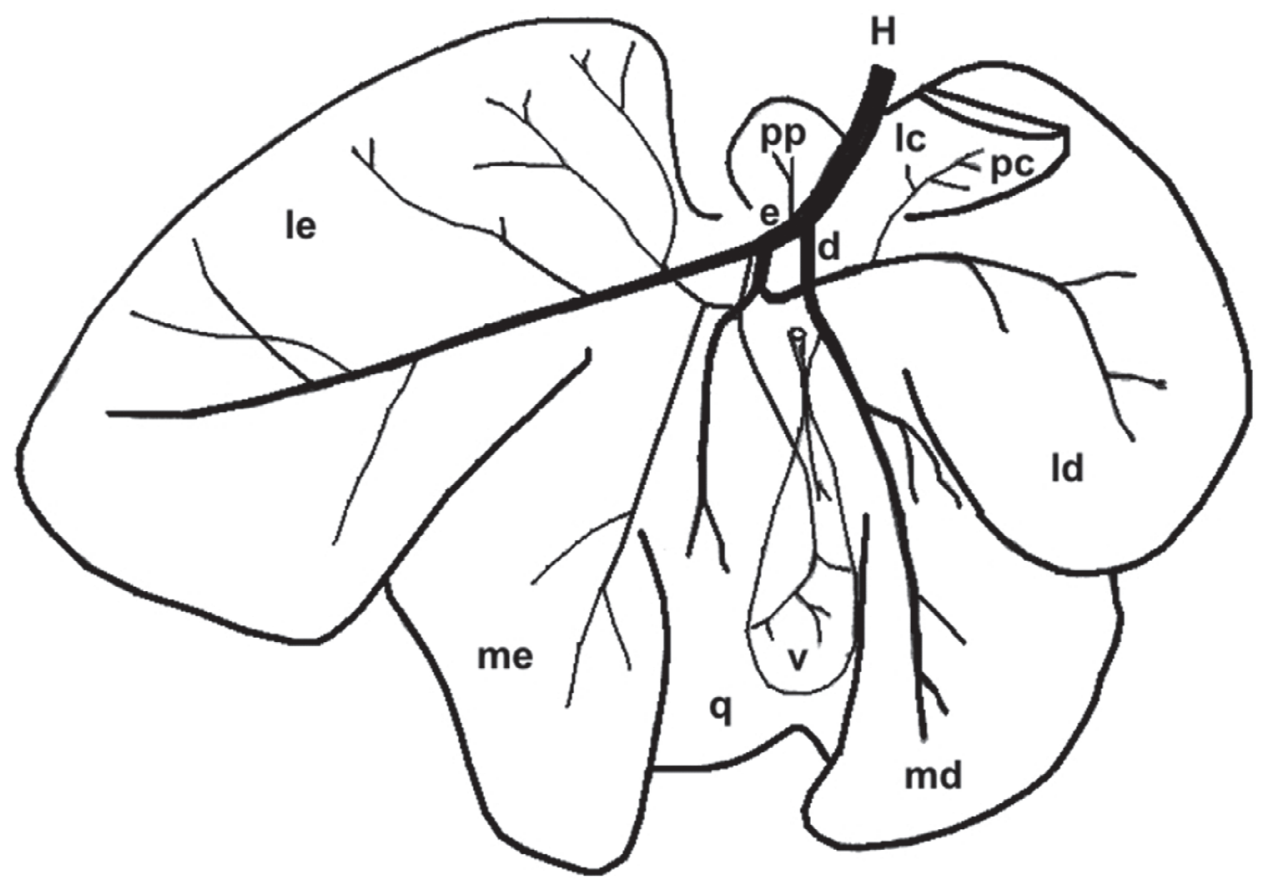

Figura 1 - Representação da artéria hepática $(\mathrm{H})$ no fígado de cutia (Dasyprocta sp.), face visceral, mostrando os ramos: direito (d), fornecendo um vaso para o lobo medial direito (md); e esquerdo (e) emitindo ramos para o lobo lateral esquerdo (le), medial esquerdo (me), lateral direito (ld), processos papilar (pp) e caudado (pc) do lobo caudado (lc), e quadrado (q). Nota-se, ainda, a vesícula biliar (v)

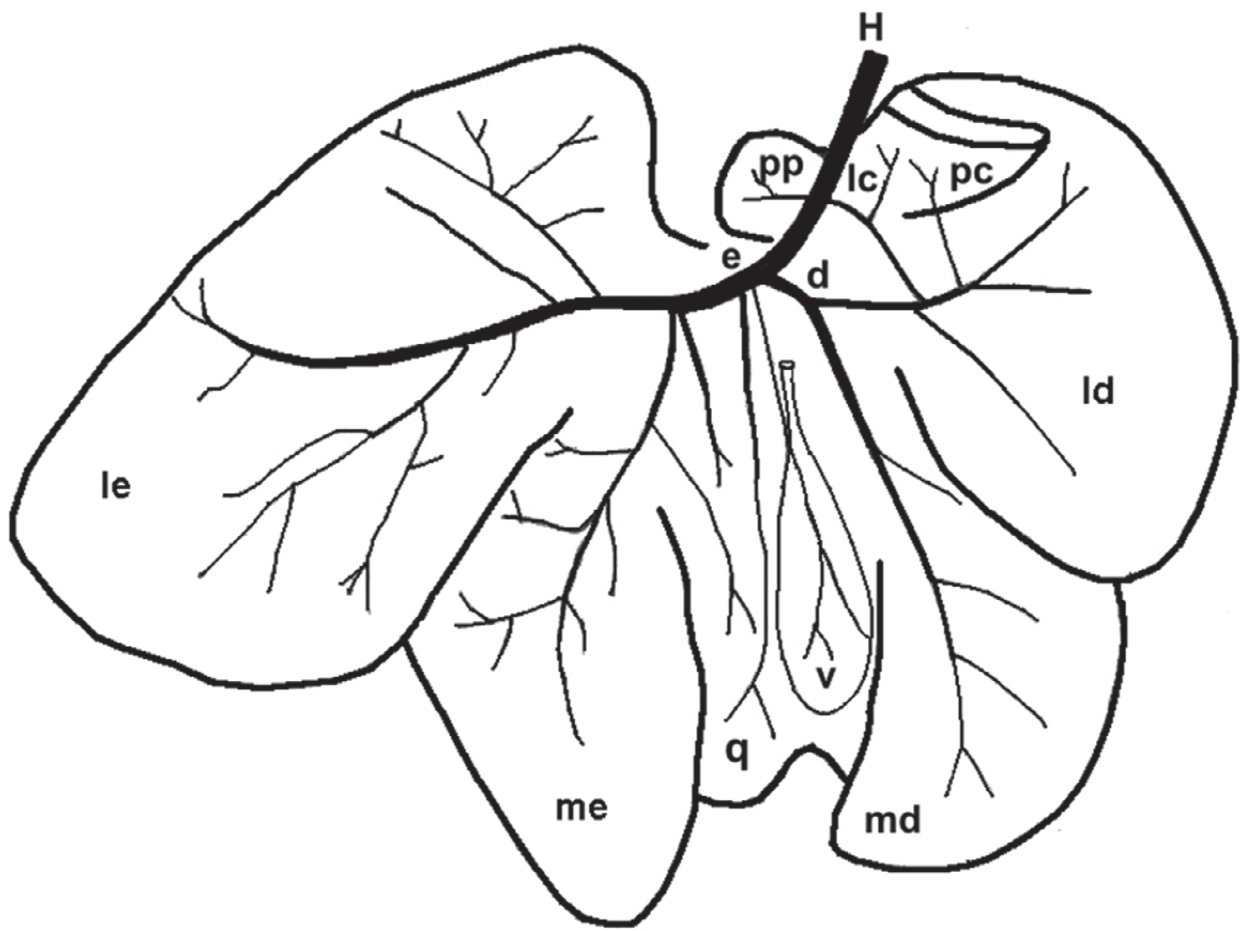

Figura 2 - Representação da artéria hepática (H) no fígado da cutia (Dasyprocta sp.), face visceral, mostrando os ramos: direito (d), fornecendo vasos para os lobos medial direito (md), lateral direito (ld) e aos processos papilar (pp) e caudado (pc) do lobo caudado (lc); o ramo esquerdo (e), para os lobos lateral esquerdo (le), medial esquerdo (me) e quadrado (q). Nota-se, ainda, a vesícula biliar (v) 


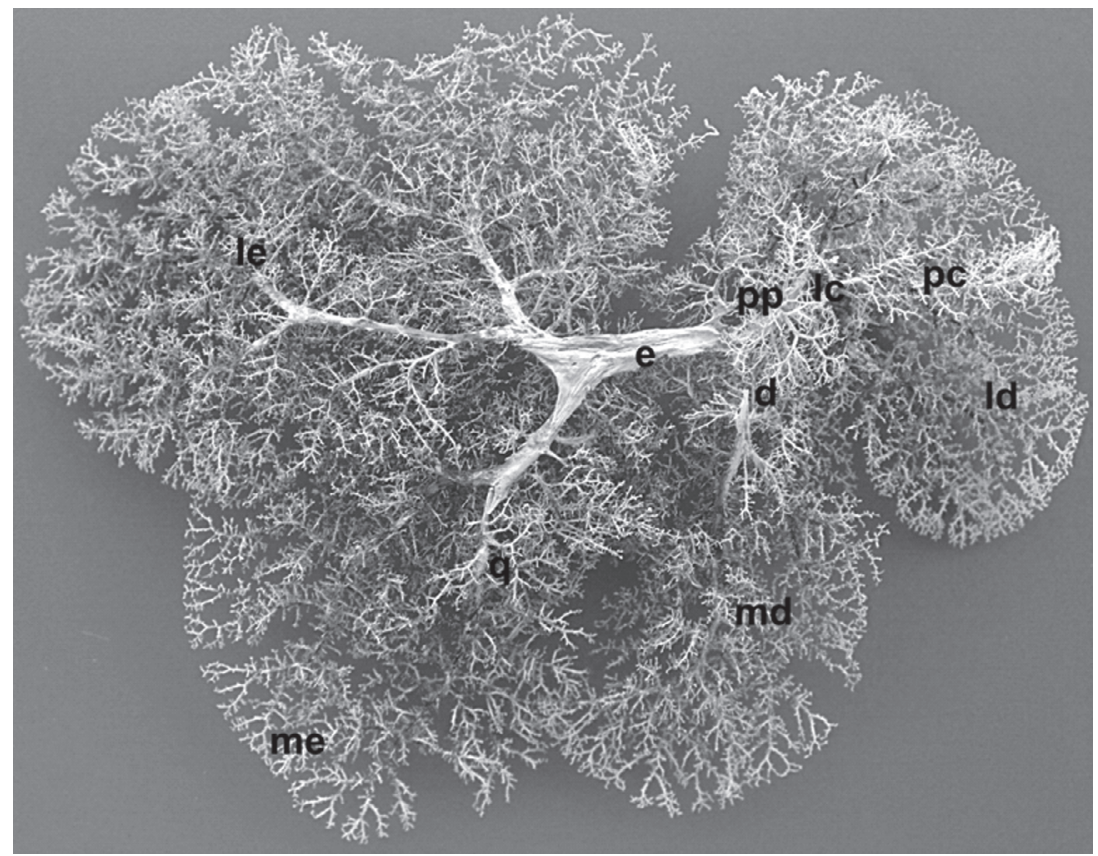

Figura 3 - Molde arterial do fígado de cutia (Dasyprocta sp.), face visceral, onde se notam os ramos: direito (d), cedendo vasos aos lobos medial direito (md), lateral direito (ld), processo caudado (pc) e processo papilar (pp) do lobo caudado (lc); o ramo esquerdo (e) fornecendo vasos aos lobos lateral esquerdo (le), medial esquerdo (me) e quadrado (q)

al. ${ }^{1}$ em coelhos. Entretanto, diferem do fígado de capivara em relação ao vaso destinado ao lobo medial direito, pois este se originava do ramo esquerdo em $66,6 \%$ dos casos de capivaras estudados por Souza et al. ${ }^{12}$. Na cutia, este ramo deu origem a vasos destinados ao lobo caudado em $80 \%$ das amostras, equivalente ao descrito na capivara $^{12}$ e em $36,7 \%$ dos casos no homem $^{13}$. Em 20\% das cutias, originava um único vaso, destinado ao lobo medial direito.

Quanto ao vaso destinado ao lobo lateral direito, ao penetrar no parênquima do hepático, na cutia, cedia, em $80 \%$, um ramo que se dirigia para a porção ventral daquele lobo, continuando-se como um tronco que se distribuia pelas porções média e dorsal. Originava, também, um único elemento ao lobo medial direito, o qual se distribuia por todo o lobo (Figura 2).

No entanto, para o lobo medial direito, o ramo principal direito fornecia, em $60 \%$, quatro vasos que se distribuíam pelo parênquima hepático e, em $40 \%$, emitia cinco ramos (Figura 2). No que se refere ao lobo caudado, este recebia vascularização do ramo direito através de vasos que se dirigiam ao processo papilar (20\%), para o processo caudado $(10 \%)$ ou tanto para o processo papilar como para o caudado $(50 \%)$.

$\mathrm{Em} 80 \%$ das cutias, o ramo esquerdo constituía um tronco comum aos vasos que se destinavam aos lobos lateral esquerdo, medial esquerdo, quadrado e, em $50 \%$, ao caudado (Figura 3). Estes achados aproximam-se dos encontrados em caprinos $^{10}$, capivaras $^{12}$ e coelhos ${ }^{1}$. Em 20\% das cutias, o ramo esquerdo emitia um ramo destinado ao processo papilar do lobo caudado e, em seguida, vasos para os lobos lateral esquerdo, medial esquerdo, quadrado e lateral direito (Figura 1).

Quanto à distribuição do ramo esquerdo, o vaso destinado ao lobo lateral esquerdo, ao penetrar no parênquima emitia, em $90 \%$ dos casos, um vaso destinado a porção dorsal, prosseguindo como um tronco que se distribuia pela porção média e ventral do lobo (Figura 2); em 10\%, fornecia um vaso para a porção dorsal e, em seguida para as porções ventral e média. Em $90 \%$ das peças analisadas o ramo 
esquerdo originava um único ramo com distribuição por todo o lobo medial esquerdo, sendo este lobo suprido por seis vasos em $60 \%$ e por três em $30 \%$. Em 20\%, o lobo lateral direito recebia vasos do ramo esquerdo, sendo um deles destinado à porção ventral e um outro para as porções média e dorsal.

Ainda em relação ao ramo esquerdo da artéria hepática, em 50\% dos casos emitia três vasos com distribuição para o parênquima do lobo quadrado e, em 40\%, fornecia apenas dois. O lobo caudado recebia suprimento sanguíneo através deste ramo para o processo papilar (10\%), para o caudado $(20 \%)$ ou para os processos papilar e caudado $(10 \%)$.

A vesícula biliar recebia suprimento arterial através do ramo esquerdo $(62,5 \%)$, do ramo direito $(25 \%)$ ou do direito e esquerdo (12,5\%). Em 10\% dos casos, foi visto uma anastomose entre o vaso que se dirigia ao processo caudado do lobo caudado e ao destinado ao lobo lateral direito.

\section{Conclusões}

1. A artéria hepática nas cutias apresenta-se dividida quase que exclusivamente $(100 \%)$ nos ramos direito e esquerdo, os quais se distribuem pelo parênquima dos lobos do fígado;

2. Com base no estudo da distribuição intraparenquimal da artéria hepática nestes roedores, pode-se inferir a presença de territórios com vascularização arterial própria nos lobos hepáticos, caracterizando, portanto, a segmentação anátomo-cirúrgica arterial.

\section{Intraparenchymal distribution of the hepatic artery in agouti (Dasyprocta sp, Rodentia)}

\begin{abstract}
The present study was design to investigate the hepatic arteries branches and your intraparenchymal distribution in agoutis. Were studied ten agouti's livers, female and male from our colony (Núcleo de Estudos de Preservação de Animais Silvestres do Centro de Ciências Agrárias da Universidade Federal do Piauí - FUFPI/IBAMA 02/99). After injection with colored latex Neoprene 650 (DuPont do Brasil, Chemistry Industries), through the hepatic artery, eight livers were dissected through the visceral faces. The samples were fixed in 10\% aqueous formaldehyde solution and after a minimum period of 48 hours. Two organs were injected with colored vinil acetate, following procedures of acid chloride corrosion in order to obtained vascular casts. The study shows that the agouti's hepatic arteries bifurcate into two principals branches, right and left (100\%). In general, the right branches usually $(80 \%)$ gave origin vessels that are responsible for the right medial, right lateral and caudate lobes's vascularization. The left branches normally $(80 \%)$ give origin vessels that are responsible for the left medial, left lateral, quadrate lobes's vascularization, also to the caudate lobos (50\%) and right lateral (20\%). In this way, the vascular distribution of the hepatic artery is related to the organ's lobos; where the right and left branches have intraparenchymal distribution into the liver's lobos. This study gave support to the anatomic-surgery artery segmentation.
\end{abstract}

Key words: Anatomy. Hepatic artery. Liver. Agouti. Rodentia. 


\section{Referências}

1 BARONE, R. et al. Atlas d'anatomie du lapin. Paris: Masson, 1973. 219 p.

2 CAVALCANTE, R. R. Digestibilidade aparente de nutrientes de rações balanceadas com alimentos alternativos com cutia Dasyprocta prymnolopha (Wagler, 1831) em crescimento. 2003. 64 f. Dissertação (Mestrado) - Centro de Ciências Agrárias, Universidade Federal do Piauí, Teresina, 2003

3 PINHEIRO, M. J. P.; ANDRADE, S. A.; CUNHA, J. N. Preservação e exploração de animais silvestres e nativos: Preá, cutia e mocó. Caatinga, n. 6, p. 28-49, 1989.

4 MENEZES, D. J. A. et al. Configuração do sistema venoso portal na cutia (Dasyprocta agut, RODENTIA). Brazilian Journal of Veterinary Reserch and Animal Science, v. 38, n. 6, p. 263-266, 2001.

5 MENEZES, D. J. A. et al. Distribuição intraparenquimal da veia porta hepática na cutia (Dasyprocta aguti RODENTIA: MAMMALIA), Archives of Veterinary Science, v. 6, n. 2, p. 1-7, 2001.

6 CARVALHO, M. A. M.; MIGLINO, M. A.; DIDIO, L. J. A. Ramificação e distribuição da artéria celíaca na cutia (Dasyprocta aguti). Brazilian Journal of Veterinary Research and Animal Science, v. 31, n. 3/4, p. 191197, 1994

7 CHIASSON, R. B. Laboratory anatomy of the white rat. 2.ed. Arizona: Brown, 1969. $81 \mathrm{p}$.

8 COOK, M. J. The anatomy of the laboratory mouse. London: Academic Press, 1965. 143 p.

9 GREENE, E. C. Anatomy of the rat. Philadelphia: American Philosophical Society, 1963. v. 2. p. $227-$ 228.

10 FRANCO, W. A. et al. Vascularização arterial do fígado em caprinos. In: CONGRESSO BRASILEIRO DE ANATOMIA, 17., 1996, Fortaleza. Anais... Fortaleza: Congresso Brasileiro de Anatomia, 1996. p. 9.

11 SOUZA, W. M.; SOUZA, N. T. M.; MIGLINO, M A. The hepatic artery in liver of Capybara (Hydrochaeris hydrochaeris). In: CONGRESSO PANAMERICANO DE ANATOMIA, 12; CONGRESSO BRASILEIRO DE ANATOMIA, 18., 1988, Navio Funchal. Anais.. Navio Funchal: Congresso Brasileiro de Anatomia, 1998. p. 12.

12 SOUZA, W. M. et al. The hepatic artery in liver of Capivara (Hydrochaeris hydrochaeris). Brazilian Journal of Morphological Sciences, v. 18, p. 182, 2001.

13 WAFAE, N.; VIEIRA, M. C.; CROZERA, Y. Ramos arteriais para o lobo caudado do fígado humano. Brazilian Journal of Morphological Sciences, v. 5, n. 2, p. 109-112, 1988 